\title{
A Schreier theorem for free topological groups
}

\section{Peter Nickolas}

\begin{abstract}
M. I. Graev has shown that subgroups of free topological groups need not be free. Brown and Hardy, however, have proved that any open subgroup of the free topological group on a $k_{\omega}$-space is again a free topological group: indeed, this is true for any closed subgroup for which a Schreier transversal can be chosen continuousiy. This note provides a proof of this result more direct than that of Brown and Hardy. An example is also given to show that the condition stated in the theorem is not a necessary condition for freeness of a subgroup. Finally, a sharpened version of a particular case of the theorem is obtained, and is applied to the preceding example.
\end{abstract}

The well-known Niel sen-Schreier theorem [4] states that every subgroup of a free group is free. Examples have been given, however, to show that the analogous statement for subgroups of a free topological group is false ([3] and [1]). Nevertheless, Brown and Hardy have proved in [2] (see also [5]) that a closed subgroup of the free topological group on a $k_{\omega}$-space will again be free, provided that a Schreier transversal for the subgroup can be chosen continuously. (Two immediate corollaries deserve mention. Firstly, any open subgroup satisfies the condition and is therefore free.

Received 9 May 1975. Communicated by Sidney A. Morris. The author is grateful to Professors Sidney A. Morris and Ronald Brown and to Dr J.P.I. Hardy for helpful comments, and to the University College of North Wales, Bangor, where this work was done. The author also acknowledges the financial assistance of an Australian Government Postgraduate Research Award. 
Seconaly, if a topological group $G$ is a $k_{\omega}$-space, then the canonical quotient from the free topological group on $G$ onto $G$ has a free topological group as its kernel.)

Brown and Hardy proved their result by means of the theory of topological groupoids ([2], [5]; see also [6]) and their techniques also yielded an open subgroup version of the Kurosh subgroup theorem for free products of topological groups. Our main aim in this note is to provide a short proof of their topological Nielsen-Schreier theorem, without recourse to the theory of topological groupoids. We then discuss an example of a free topological group with a subgroup which is free, but for which a Schreier transversal cannot be chosen continuously. A sharpened version of the theorem in a special case is then proved, and applied to the preceding example.

We now list a few facts, and establish the notation to be used later. We assume the reader to be familiar with the concept of the free topological group on a pointed space, as defined by Graev [3]. (See also [9] and [7].)

Recall that a Hausdorff space $X$ is a $k_{\omega}$-space if it is the union of an increasing sequence $\left\{x_{n}\right\}$ of compact sets, with the property that a set $A \subseteq X$ is closed if and only if $A \cap X_{n}$ is compact for each $n$. (We refer to $\mathrm{UX}_{n}$ as the decomposition of $X$.) Any compact set in such a space lies in some $X_{n}$. For this and further information, see [7] and [8].

If $Y$ is a subset of a group $G$, we denote by $g p(Y)$ the subgroup of $G$ generated by $Y$, and by $g p_{n}(Y)$ those elements of $g p(Y)$ which can be written as words of lengths less than or equal to $n$ with respect to $Y$. In particular, if $F(X)$ is the free topological group on a pointed space $X$, we shall write $F_{n}(X)$ for $\operatorname{gP}_{n}(X)$; and if $X$ is a $k_{\omega}$-space with decomposition $\mathrm{U} X_{n}$, we shall write $F_{n}\left(X_{n}\right)$ for $\mathrm{gp}_{n}\left(x_{n}\right)$.

With this notation, Theorem 1 of [7] shows that if $X$ is a $k_{\omega}$-space as above, then $F(X)$ is also a $k_{\omega}$-space, with decomposition $U F_{n}\left(X_{n}\right)$. 
Theorem 3 of [7] gives conditions under which a subgroup of a free topological group is again free. We begin with a slightly sharpened version of this result.

THEOREM 1. Let $X$ be a $k_{\omega}$-space with decomposition $U X_{n}$, and let $Y$ be a subspace of $F(X)$ such that $Y \backslash\{e\}$ freely generates $g p(Y)$. Then the following are equivalent:

(1) $Y$ is the zonion of an increasing sequence of compact sets $\left\{y_{n}\right\}$, such that for each $n$ there exists an $m$ for which $\operatorname{gp}(Y) \cap F_{n}\left(X_{n}\right) \subseteq \mathrm{gp}_{m}\left(Y_{m}\right) ;$

(2) there is a decomposition $U Y_{n}$ of $Y$ as a $k_{\omega}$-space, such that for each $n$ there exists an $m$ for which $\mathrm{gp}(Y) \cap F_{n}\left(X_{n}\right) \subseteq \mathrm{gp}_{m}\left(Y_{m}\right)$;

(3) $\mathrm{gp}(Y)$ is $F(Y)$, and $\mathrm{gp}(Y)$ and $Y$ are closed in $F(X)$.

Proof. That (2) implies (3) is Theorem 3 of [7], and that (2) implies (I) is trivial.

Suppose (I) holds: we shall show that the given union $Y=U Y_{n}$ is a decomposition of $Y$ as a $k_{\omega}$-space (cf. Theorem 4 of [7]). Now

$$
\begin{aligned}
Y \cap F_{n}\left(X_{n}\right) & =Y \cap \operatorname{gp}(Y) \cap F_{n}\left(X_{n}\right) \\
& \subseteq Y \cap \mathrm{gp}_{m}\left(Y_{m}\right) \quad(\text { for some } m) \\
& =Y_{m} .
\end{aligned}
$$

Therefore $Y \cap F_{n}\left(X_{n}\right) \subseteq Y_{m} \cap F_{n}\left(X_{n}\right)$, and since $Y_{m} \subseteq Y$, we have $Y \cap F_{n}\left(X_{n}\right)=Y_{m} \cap F_{n}\left(X_{n}\right)$. But both $Y_{m}$ and $F_{n}\left(X_{n}\right)$ are compact, and so $Y \cap F_{n}\left(X_{n}\right)$ is compact. Since this holds for each $n$, and $F(X)=U F_{n}\left(X_{n}\right)$ is a $k_{\omega}$-space, $Y$ is closed in $F(X)$. Hence $Y$ is a $k_{\omega}$-space with decomposition $U\left(Y \cap F_{n}\left(X_{n}\right)\right)$. To show that $U Y_{n}$ is also such a decomposition, we need only find for each $n$ an $m$ for which $Y \cap F_{n}\left(X_{n}\right) \subseteq Y_{m}$ (see $\S 2$ of [7]); and this we have already done. Thus (2) is proved. 
Suppose now that (3) holds. If we set $Y_{n}=Y \cap F_{n}\left(x_{n}\right)$, the fact that $Y$ is closed implies that $U Y_{n}$ is a decomposition of $Y$ as a $k_{\omega}$-space. By Theorem 1 of [7], $g p(Y)=F(Y)$ is then a $k_{\omega}$-space, with decomposition $U_{g p_{m}}\left(Y_{m}\right)$. But $g P(Y)$ is closed in $F(X)$, and so $\operatorname{gp}(Y) \cap F_{n}\left(X_{n}\right)$ is compact for each $n$, and there is an $m$ such that $\mathrm{gp}(Y) \cap F_{n}\left(X_{n}\right) \subseteq \mathrm{gP}_{m}\left(Y_{m}\right)$. Therefore (2) holds, completing the proof.

We now prove the topological Nielsen-Schreier theorem of Brown and Hardy.

THEOREM 2 [2]. Let $G=F(X)$ be the free topological group on a $k_{\omega}$-space $X=U X_{n}$, and let $H$ be a subgroup of $G$. Suppose that the projection $p$ from $G$ onto $G / H$ (the space of right cosets of $H$ ) has a continuous section $s: G / H \rightarrow G$ such that $s(G / H)$ is a Schreier transversal for $H$ in $G$. Then $H$ is closed and is a free topological group.

Proof. If we set $B=\left\{s(H g)_{x s}(H g x)^{-1}: g \in G, x \in X\right\}$, the usual proof of the Nielsen-Schreier theorem (see [4]) shows that $B \backslash\{e\}$ is algebraically a free basis for $H$.

Define $\phi: G \times X \rightarrow G$ by $\phi:(g, x) \mapsto s(H g)_{x s}(H g x)^{-1}$, for $g \in G$ $x \in X$. Clearly $B=\phi(G \times X)$. Now each of the following functions is continuous on $G \times X$ :

$$
\begin{aligned}
& (g, x) \mapsto g \mapsto p(g)=H g \mapsto s(H g), \\
& (g, x) \mapsto x, \\
& (g, x) \mapsto g x \mapsto p(g x)=H g x \mapsto s(H g x) ;
\end{aligned}
$$

and thus $\phi$ is itself continuous, by the continuity of multiplication and inversion in $G$. Defining $B_{n}$ to be $\phi\left(E_{n}\left(X_{n}\right) \times X_{n}\right)$ we see that each $B_{n}$ is compact, that $B_{1} \subseteq B_{2} \subseteq \cdots$, and that $B=\bigcup_{n=1}^{\infty} B_{n}$. According to the previous theorem, to show that $H$ is $F(B)$ and is closed in $G$ we need only find for each $n$ an $m$ for which $H \cap F_{n}\left(X_{n}\right) \subseteq \operatorname{gp}_{m}\left(B_{m}\right)$. 
To this end, let $h \in H \cap F_{n}\left(X_{n}\right)$. Then $h$ can be written in reduced

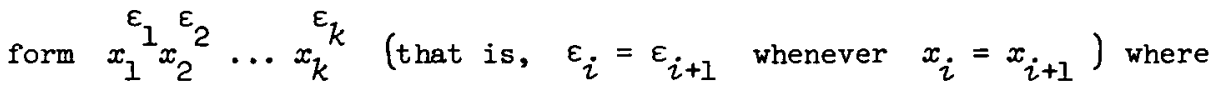
$k \leq n$ and $x_{i} \in X_{n}$ for $i=1,2, \ldots, k$. Since $h \in H$, the proof of the Nielsen-Schreier theorem shows that we can also write $h=c_{1} c_{2} \cdots c_{k}$, where

$$
c_{i}=s\left(H x_{1}^{\varepsilon_{1}}{ }_{x_{2}}^{\varepsilon_{2}} \ldots x_{i-1}^{\varepsilon_{i-1}}\right) x_{i}^{\varepsilon_{i}}\left(H x_{1}^{{ }^{1}}{ }_{x_{2}}^{\varepsilon_{2}} \ldots x_{i-1}^{\varepsilon_{i-1} x_{i}^{\varepsilon_{i}}}\right)^{-1} \text {, }
$$

for $i=1,2, \ldots, k$. Now it is clear that for each $i \leq k$,

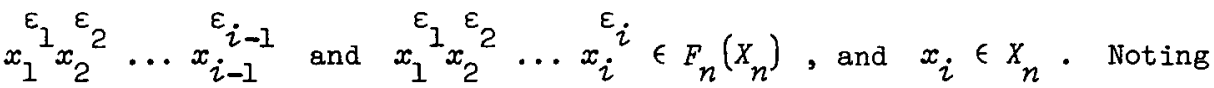
that if $\varepsilon_{i}=1$ then $c_{i}=\phi\left(x_{1}{ }^{{ }_{1}}{ }^{\varepsilon_{2}}{ }_{2} \ldots x_{i-1}^{\varepsilon_{i-1}}, x_{i}\right)$, we see that $c_{i} \in \phi\left(F_{n}\left(X_{n}\right) \times X_{n}\right)=B_{n}$. Similarly, if $\varepsilon_{i}=-1$, then $c_{i}^{-1}=\phi\left(x_{1}^{\varepsilon_{1}{ }^{1}{ }_{2}{ }_{2}} \ldots x_{i}^{\varepsilon_{i}}, x_{i}\right) \in \phi\left(F_{n}\left(x_{n}\right) \times x_{n}\right)=B_{n}$. Hence $h=c_{1} c_{2} \cdots c_{k} \in \operatorname{gp}_{n}\left(B_{n}\right)$, since $k \leq n$; that is, $H \cap F_{n}\left(X_{n}\right) \subseteq \operatorname{gp}_{n}\left(B_{n}\right)$ for all $n$. This proves the theorem.

The following example shows that the condition given in Theorem 2 for a subgroup of $F(X)$ to be free is not a necessary condition. Let $X$ be a compact Hausdorff space, and $Y$ a closed subset of $X$ containing $e$, the base-point of $X$. By Theorem 1 , the subgroup $H=g p(Y)$ of $F(X)$ is $F(Y)$. Suppose that there exists a continuous section $s$ of the projection $p$ as in Theorem 2. Let $x \in X \backslash Y$ and suppose that

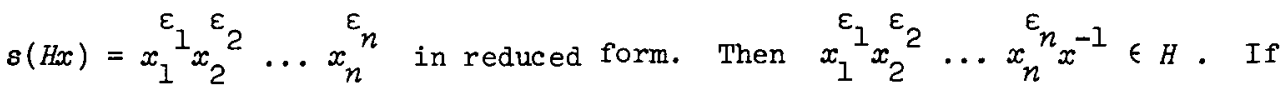
$x_{n}^{\varepsilon_{n}} \neq x$, then $x_{1}^{\varepsilon_{1}} x_{2}^{\varepsilon_{2}} \ldots x_{n}^{\varepsilon_{n}}{ }^{-1}$ is reduced (as written) with respect to $X$, and therefore with respect to $Y$, and so $x_{1}, x_{2}, \ldots, x_{n}, x \in Y$, contradicting $x \in X \backslash Y$. We must therefore have $x_{n}^{\varepsilon_{n}}=x$. But this implies that $x_{1}^{\varepsilon_{1}} x_{1}^{\varepsilon_{2}} \ldots x_{n-1}^{\varepsilon_{n-1}} \in H$, and since we already know that 
$x_{1}^{E_{1}} x_{2}^{\varepsilon_{2}} \ldots x_{n-1}^{\varepsilon_{n-1}}$ is in the Schreier transversal for $H$ (because it is an initial segment of $x_{1}^{\varepsilon_{1}}{ }^{\varepsilon_{2}} \ldots x_{n}^{\varepsilon_{n}}$ ), we have ${ }_{x_{1}}^{\varepsilon_{1}{ }^{\varepsilon_{2}}{ }_{2}} \ldots x_{n-1}^{\varepsilon_{n-1}}=e$; that is, $s(H x)=x_{n}^{\varepsilon_{n}}=x$, for $x \in X \backslash Y$. For $x \in Y, x \in H$ also, and thus $\boldsymbol{s}(H x)=e$. Since $X$ is compact, and $s$ and $p$ are continuous, we find that $s(p(X))=X \backslash Y \cup\{e\}$ is compact. Taking $X=[0,1], Y=\left[0, \frac{7}{2}\right]$, and $e=0$, for example, we have $X \backslash Y \cup\{e\}=\{0\} \cup\left(\frac{1}{2}, 1\right]$, which is certainly not compact.

The above paragraph describes a situation in which Theorem 2 does not apply. Under some circumstances, however, a result somewhat stronger can be obtained from the proof of Theorem 2.

COROLLARY. Let $X=U X_{n}$ be a $k_{\omega}$-space and let $H$ be a subgroup of $G=F(X)$. Suppose that there is a compact set $Y$ in $H$ such that $Y \backslash\{e\}$ is algebraically a free basis for $H$, and that $Y \backslash\{e\}$ can be obtained as a free basis from some schreier transversal for $H$ in $G$. Then $H$ is $F(Y)$ and is closed in $G$.

Proof. By Theorem $I$ we have only to show that for each $n$ there is an $m$ such that $H \cap F_{n}\left(X_{n}\right) \subseteq \mathrm{gp}_{m}(Y)$. This statement is proved exactly as the corresponding statement was proved in Theorem 2, except that the functions $s$ and $\phi$ need no longer be continuous.

If we now return to our example above, the Corollary shows easily that $H=F(Y)$. A Schreier transversal for $H$ in $F(X)$ certainly exists (it may be constructed in the usual inductive way [4]). If $r(g)$ denotes the representative of an element $g \in F(X)$, the free basis defined by the Schreier transversal contains the elements $r(e) y r(y)^{-1}$ for each $y \in Y$. But since $y \in H, r(e)=r(y)=e$, and so the free basis contains $Y$ : because $Y$ is already a free basis however, the new basis must be precisely $Y$. Thus the hypotheses of the Corollary are satisfied, and $H=F(Y)$. 


\section{References}

[1] Ronald Brown, "Some non-projective subgroups of free topological groups", Proc. Amer. Math. Soc. (to appear).

[2] R. Brown and J.P.L. Hardy, "Subgroups of free topological groups and free topological products of topological groups", J. London Math. Soc. (to appear).

[3] M.1. Graev, "Free topological groups", Amer. Math. Soc. Transz. 35 (1951), 61 pp.; reprinted Amer. Math. Soc. Transl. (1) 8 (1962), $305-364$.

[4] Marshall Hall, Jr., The theory of groups (Macmillan, New York, 1959).

[5] J.P.L. Hardy, "Topological groupoids: coverings and universal constructions", ( $\mathrm{PhD}$ thesis, University College of North Wales, Bangor, Wales, 1974).

[6] Philip J. Higgins, Notes on categories and groupoids (Van Nostrand Reinhold, London, New York, Cincinnati, Toronto, Melbourne, 1971).

[7] J. Mack, Sidney A. Morris and E.T. Ordman, "Free topological groups and the projective dimension of a locally compact abelian group", Proc. Amer. Math. Soc. 40 (1973), 303-308.

[8] Bernard L. Madison, "Congruences in topological semigroups", Second Florida Symposium on automata and semigroups (University of Florida, Tampa, 1971).

[9] A.A. Markov, "On free topological groups", Amer. Math. Soc. Transl. 30 (1950), 11-88; reprinted Amer. Math. Soc. Transl. (1) 8 (1962), 195-272.

School of Mathematics,

University of New South Wales,

Kensington,

New South Wales. 